\title{
CNS MALFORMATIONS
}

\section{COWDEN SYNDROME WITH CORTICAL MALFORMATION AND EPILEPSY}

Investigators at the Institute for Research on Mental Retardation and Brain Aging, Troina, and University of Naples, Italy report a case of Cowden syndrome presenting with unilateral perisylvian dysplasia and with drug resistant focal seizures. A 14-year-old girl was born with hemiparesis and at 4 months she presented with seizures. Birth weight, height, and head circumference were above the $90^{\text {th }}$ centile. MRI showed a dysplastic cleft and polymicrogyria in the right Sylvian region. The left cerebellar hemisphere was enlarged with dysplastic and hamartomatous appearance characteristic of LhermitteDuclos disease, part of the Cowden syndrome. At 7 years of age she developed obstructive hydrocephalus with herniation of cerebellar tonsils, relieved by a VP shunt and removal of the cerebellar hamartoma, histologically a cerebellar gangliocytoma. Seizures involving left side were refractory to medications. Intestinal examination showed small duodenal polyps. PTEN sequence analysis showed a de novo missense mutation. (Elia M, Amato C, Bottitta M, et al. An atypical patient with Cowden syndrome and PTEN gene mutation presenting with cortical malformation and focal epilepsy. Brain Dev 2012 Nov;34(10):873-6). (Respond: Dr Marco Carotenuto, Second University of Naples, Napoli, Italy. E-mail: marco.carotenuto@unina2.it).

COMMENT. Cowden (or multiple hamartoma) syndrome, named after the first patient reported (Lloyd KM, Dennis M. Ann Intern Med 1963 Jan;58:136-42), is characterized by macrocephaly, intestinal hamartomatous polyps, benign skin tumors, and dysplastic gangliocytoma of the cerebellum (Lhermitte-Duclos disease). The occurrence of drug resistant epilepsy with Cowden syndrome is explained by an associated cortical dysplasia.

\section{EPIDERMAL NEVUS SYNDROME ASSOCIATED WITH BRAIN MALFORMATIONS AND MEDULLOBLASTOMA}

Researchers at Juntendo University and Tokyo Women's Medical University, Japan; and University of California, San Francisco, Ca, report a male infant with epidermal nevus syndrome associated with brainstem and cerebellar malformations and neonatal medulloblastoma. Macrocephaly and enlarged fourth ventricle were noted on fetal ultrasound. At birth the patient had epidermal nevi, brain malformations including polymicrogyria, dysmorphic and enlarged midbrain tectum, and enlarged cerebellar hemispheres. The patient died after surgical resection of a medulloblastoma, diagnosed on MRI at 51 days of age. At autopsy, the cerebellum had many foci of heterotopia and the brainstem showed multiple anomalies, including enlarged superior colliculi, hypoplastic pyramidal tracts and dysplasia of inferior olivary nuclei. These malformations extend the spectrum of epidermal nevus syndrome. (Okumura A, Lee T, Ikeno $\mathrm{M}$, et al. A severe form of epidermal nevus syndrome associated with brainstem and cerebellar malformations and neonatal medulloblastoma. Brain Dev 2012 Nov;34(10):881-5). (Respond: Dr Akihisa Okumura, Department of Pediatrics, Juntendo University Faculty of Medicine, Tokyo, Japan. E-mail: okumura@juntendo.ac.jp). 
COMMENT. Epidermal nevus syndrome (or Solomon syndrome) is characterized by various epidermal nevi, including ichthyosis, acanthotic, and sebaceous, mental retardation, epilepsy, ocular abnormalities, including coloboma, microphthalmos, cataracts, and skeletal, cardiac, and urogenital abnormalities. Associated abnormalities include hemihypertrophy, hemimegalencephaly, seizures, including infantile spasms, sensorineural deafness, spastic hemiparesis, kyphoscoliosis, and polydactyly. A tendency to malignant transformation of nevi and associated visceral malignancies (Wilms tumor, astrocytoma, intrathoracic teratoma) are reported. (Egan CA, et al. Neurologic variant of epidermal nevus syndrome with a facial lipoma. Int J Dermatol 2001 Mar;40(3):18990).

\section{CNS NEOPLASMS}

\section{CLINICO-RADIOLOGICAL PROFILE OF PEDIATRIC GLIOBLASTOMA}

Researchers at Sanjay Gandhi Post Graduate Institute of Medical Sciences, Lucknow, India, studied the clinico-radiological profile, pathology, treatment and outcome of 65 pediatric patients (age $<18$ years) with histopathologically proven diagnosis of intracranial glioblastoma. Male-to-female ratio was 2.6:1, with a mean age at diagnosis of 13.3 years (range 2-18 years). Most common presenting symptoms were headache with or without vomiting $(n=51,78 \%)$, seizures $(n=42,65 \%)$, and focal deficits $(\mathrm{n}=31,47 \%)$. The tumor was supratentorial in $62(95.4 \%)$ patients, frontal in $30 \%$, and temporal in $9 \%$; it was located deeply in $16(25 \%)$, in the thalamus in $10(12 \%)$. Obstructive hydrocephalus occurred in $13(20 \%)$ patients and intratumoral bleeding in 5 (7.7\%). Total tumor excision was achieved in $43(66 \%)$ patients, and the remainder had incomplete excisions $(\mathrm{n}=22,34 \%)$. Mean progression-free and overall survivals were 10 and 20 months, respectively; 3 patients survived for $>5$ years. Extent of resection was the independent predictor of survival $(\mathrm{p}=0.002)$. (Das KK, Mehrotra A, Nair AP, et al. Pediatric glioblastoma: clinico-radiological profile and factors affecting the outcome. Childs Nerv Syst 2012 Dec;28(12):2055-62). (Response: Dr Raj Kumar. E-mail: rajkumar1959@gmail.com).

COMMENT. Glioblastoma is an uncommon brain tumor in children compared to the prevalence in adults. Of all CNS tumors in children glioblastoma accounts for $\sim 3-$ $9 \%$; the higher figure is that of Bailey $\mathrm{P}$, Buchanan DN, and Bucy PC, in their classic study in Chicago (Bailey P, Buchanan DN, Bucy PC. Intracranial Tumors of Infancy and Childhood. Chicago: University of Chicago Press; 1939). The relative frequencies of the pathological varieties of intracranial space occupying lesions in children have changed over time; almost a century ago, tuberculoma was the most common lesion (Critchley M. Brain tumours in children: Their general symptomatology. Br J Child Dis $1925 ; 22: 251-264)$.

The prevalence estimates for primary brain tumors in the United States are 35.4 per 100,000 person-years for children $<20$ years old and 278/100,000 for adults. (Porter KR, et al. Neuro Oncol 2010 Jun;12(6):520-7). The prevalence for malignant brain tumors in children is 25/100,000 and for non-malignant tumors, 11/100,000. 